\title{
Association of NTRK3 and its interaction with NGF suggest an altered cross- regulation of the neurotrophin signaling pathway in eating disorders
}

Josep Maria, Mercader ${ }^{1,2}$; Ester, Saus ${ }^{1,2}$; Zaida, Agüera ${ }^{3}$; Mònica, Bayés ${ }^{1,2,4}$; Claudette, Boni $^{5}$; Anna, Carreras ${ }^{1,2,4}$; Elena, Cellini ${ }^{6}$; Rafael, de Cid ${ }^{1,2,4}$; Mara, Dierssen ${ }^{1,7}$;

Geòrgia, Escaramís ${ }^{2,1}$; Fernando, Fernández-Aranda ${ }^{8,3}$; Laura, Forcano ${ }^{3}$; Xavier, Gallego $^{1}$; Juan Ramón, Gonzàlez ${ }^{9,4}$; Philip, Gorwood ${ }^{5}$; Johannes, Hebebrand ${ }^{10}$; Anke, Hinney $^{10}$; Benedetta, Nacmias ${ }^{6}$; Anna, Puig ${ }^{1,2,4}$; Marta, Ribasés ${ }^{11}$; Valdo, Ricca ${ }^{6}$; Lucia, Romo $^{12}$; Sandro, Sorbi ${ }^{6}$; Audrey, Versini ${ }^{13}$; Mònica, Gratacòs ${ }^{2,1,4^{*}}$ and Xavier, Estivill ${ }^{1,2,4,14, *}$

${ }^{1}$ Genes and Disease Program, Center for Genomic Regulation (CRG), 08003 Barcelona, Catalonia, Spain

${ }^{2}$ CIBER en Epidemiología y Salud Pública (CIBERESP), 08003 Barcelona, Catalonia, Spain ${ }^{3}$ CIBER Fisiopatologia Obesidad y Nutricion (CIBEROBN), 08003 Barcelona, Catalonia, Spain ${ }^{4}$ National Center of Genotyping (CEGEN), Barcelona Node, 08003 Barcelona, Catalonia, Spain ${ }^{5}$ Hospital Louis Mourier, Pr Ades Department, 75014 Paris, France

${ }^{6}$ Department of Neurological and Psychiatric Sciences, University of Florence, 50121 Florence, Italy.

${ }^{7}$ CIBER Enfermedades Raras (CIBERER), 08003 Barcelona, Catalonia, Spain

${ }^{8}$ Department of Psychiatry, University Hospital of Bellvitge, L’Hospitalet de Llobregat, 08907 Barcelona, Catalonia, Spain

${ }^{9}$ Center for Research in Environmental Epidemiology (CREAL), 08003 Barcelona, Catalonia, Spain

${ }^{10}$ Rheinische Kliniken Essen, Department of Child and Adolescent Psychiatry, University of Duisburg-Essen, 45147 Essen, Germany

${ }^{11}$ Department of Psychiatry, Hospital Universitari Vall d'Hebron, Universitat Autonoma de Barcelona, 08035 Barcelona, Catalonia, Spain

${ }^{12}$ CMME Sainte Anne. 100 rue de la Santé. 75014 Paris, France

${ }^{13}$ INSERM U675. University Xavir Bichat (IFR02), 75018 Paris, France

${ }^{14}$ Experimental and Health Sciences Department, Pompeu Fabra University, 08003 Barcelona, Catalonia, Spain 


\section{Corresponding author:}

\section{Mònica Gratacòs}

CIBER en Epidemiología y Salud Pública (CIBERESP)

Genes \& Disease Program

Center for Genomic Regulation (CRG)

Plaça Charles Darwin s/n (Dr. Aiguader 88), PRBB Building, Room 521

08003 Barcelona, CATALUNYA, Spain

Phone: 34933160138

FAX: 34933160099

E-mail: monica.gratacos@crg.es 


\section{ABSTRACT}

Eating disorders (ED) are complex psychiatric diseases that include anorexia nervosa and bulimia nervosa, and have higher than $50 \%$ heritability. Previous studies have found association of BDNF and NTRK2 to ED, while animal models suggest that other neurotrophin genes might also be involved in eating behavior. We have performed a family based association study with 151 TagSNPs covering ten neurotrophin signaling genes: NGFB, BDNF, NTRK1, NGFR/p75, NTF4/5, NTRK2, NTF3, NTRK3, CNTF and CNTFR in 371 ED trios of Spanish, French and German origin. Besides several nominal associations, we found a strong significant association after correcting for multiple testing $\left(\mathrm{p}=1.04 \times 10^{-4}\right)$ between ED and rs7180942, located in the NTRK3 gene, which followed an overdominant model of inheritance. Interestingly, HapMap unrelated individuals carrying the rs7180942 risk genotypes for ED showed higher levels of expression of NTRK3 in lymphoblastoid cell lines. Furthermore, higher expression of the orthologous murine Ntrk3 gene was also detected in the hypothalamus of the anx/anx mouse model of anorexia. Finally, variants in NGFB gene appear to modify the risk conferred by the NTRK3 rs7180942 risk genotypes $\left(\mathrm{p}=4.0 \times 10^{-5}\right)$ showing a synergistic epistatic interaction. The reported data, in addition to the previous reported findings for BDNF and NTRK2, point neurotrophin signaling genes as key regulators of eating behavior and their altered cross-regulation as susceptibility factors for eating disorders. 


\section{Introduction}

Eating disorders (ED) are multifactorial psychiatric diseases that include anorexia nervosa (AN), bulimia nervosa (BN) and other intermediate or atypical conditions (1). During the last years, the incidence of these disorders has increased in industrialized countries and have become a growing problem, especially among adolescents (2). In the etiology of these disorders, genetic, psychological, socio-cultural, nutritional, neurochemical and hormonal factors seem to predispose, unleash or maintain the condition $(3,4)$.

Family and twin studies have estimated that 33-84\% of the variance in the predisposition to $\mathrm{AN}(5,6)$ and $58-83 \%$ to $\mathrm{BN}(7-9)$ may be genetic in nature. Furthermore, there is evidence that common etiologic features contribute to both AN and $\mathrm{BN}$, and a shared familial component between the two disorders has been described $(10-12)$.

The family of neurotrophins comprises four related proteins, namely nerve growth factor (NGFB), brain-derived neurotrophic factor (BDNF), and neurotrophins 3 and 4/5 (NTF3 and NTF4/5). The effects of neurotrophins are mediated by high affinity tyrosine kinase receptors, each preferentially activated by one or more neurotrophins: NTRK1 by NGFB, NTRK2 by BDNF and NTF4/5, and NTRK3 by NTF3. Besides them, a low-affinity pan-neurotrophin receptor (p75), a member of the tumor necrosis factor family, forms a complex with other neurotrophin receptors and modulates its signal transduction (reviewed in $(13,14))$. During development, neurotrophins play important roles in proliferation, differentiation and survival of neurons in both the central and peripheral nervous system. In adults, they control neural plasticity and regulate synaptic activity and neurotransmitter synthesis (reviewed in (15)). There is an increasing recognition that the pathophysiology of several mental disorders, including 
depression, anxiety, schizophrenia, and drug addiction, could be the result of a local deregulation of synaptic plasticity. A possible neurobiological explanation for this theory would be an altered synthesis and/or release of neurotrophins (16-19).

Since BDNF and its receptor NTRK2 have been shown to be involved in the regulation of eating behavior and energy balance $(16,20)$, we and others have studied the role of variation in these genes in ED and determined that both BDNF and NTRK2 play a role in the disease susceptibility (21-25). Furthermore, animal models suggest that other neurotrophins might also be involved in the regulation of food intake. For example, intracerebroventricular infusion of NGF causes a centrally mediated, dosedependent hypophagia and weight loss in non-obese mice (26). Dietary restriction can enhance hippocampal neurogenesis in a mouse model, resulting in an increased production of BDNF and NTF3 (27). In addition, NTF4 knockout mice exhibit deficits in feeding behavior, suggesting reduced food satiation $(28,29)$. Indeed, the infusion of NTF4/5 into the third ventricle of BDNF heterozygous mutant mice can transiently reverse its hyperphagic and obese phenotype (16). Finally, ciliary neurotrophic factor (CNTF) and it's receptor (CNTFR), have been shown to exert a neuroprotective effect in a non-human primate model (30), and CNTF has been described as a leptin-like molecule with a profound effect on appetite and energy expenditure $(31,32)$. This effect is thought to be the result of the activation of signaling cascades in hypothalamic nuclei involved in feeding control (33-35).

We hypothesized that genetic variation in one or more genes from the neurotrophic system, above and beyond BDNF and NTRK2, may lead to an abnormal modulation of feeding behavior and hence to eating disorders in humans. To test this hypothesis, we selected informative polymorphisms spanning the entire loci of p75 (NGFR), NGFB, NTRK1, BDNF, NTRK2, NTF3, NTRK3, NTF4, CNTF and CNTFR, 
and variations within each gene were analyzed in a set of 371 ED trios of Spanish, French and German origin. 


\section{Results}

Dataset consisted of 386 ED female trios from Barcelona, Paris and Marburg/Essen. Fifteen families were excluded from the analysis because they had more than three SNPs with Mendelian errors, and were thus considered false paternity. Thus, the final dataset available for the analyses was composed of 371 trios (286 AN, 59 BN and 26 EDNOS). The demographic and clinical characteristics of the samples are summarized in Table 1. A total of 183 TagSNPs were selected for extensive coverage of the genomic regions corresponding to 10 neurotrophic factor and receptor genes: $p 75, N G F B, B D N F$, NTF3, NTF4, CNTF, NTRK1, NTRK2, NTRK3 and CNTFR, which range in size from 6 to $409 \mathrm{~kb}$. Each gene was tagged with a minimum of three and a maximum of 58 TagSNPs. The complete list of the genes, their chromosomal locations, the characteristics of the TagSNPs, as well as the linkage disequilibrium (LD) structure of each gene are shown in Supplementary Table 1 and Supplementary Figures 2-10. For the analysis, we removed those SNPs, which showed less than $80 \%$ genotyping success rate $(\mathrm{n}=31)$ and one variant that deviated from Hardy-Weinberg equilibrium (alpha = 0.01), ending up with a total of 151 SNPs. Genotype concordance with respect to the HapMap reference trios was of $100 \%$.

We found no evidence of genetic heterogeneity between the three available groups of samples except for one variant in CNTFR, which was not included in the analysis. For the rest of SNPs, there were no significant differences in their allele frequencies among populations after correcting for multiple testing (Supplementary Figure 1). Thus, further analyses were performed using the combined dataset.

\section{Single marker analysis}

Family-based association tests for ED phenotype, under a codominant model, detected 14 nominal positive SNPs, although only rs7180942, located in intron 8 of NTRK3, 
reached statistical significance after Bonferroni correction $\left(Z=-3.9 ; p=1.04 \times 10^{-4}\right)$ (Supplementary Table 2 and Figure 1). When the sample was stratified by AN and BN, 11 and 12 SNPs were found to be nominally associated, respectively, although none of them remained statistically significant after correction for multiple testing (Supplementary Table 2). Interestingly, seven SNPs in NTRK3 were associated with at least one of the clinical groups tested (Figure 1).

Next, focused on NTRK3 rs7180942 and performed a stratified analysis subdividing AN between purging and non-purging subtype (ANP, or ANR), and analyzing solely BN-purging subtypes (BNP). In all disease phenotypes, the CT genotype was undertransmitted to probands, although only in the case of ED it remained statistically significant with Bonferroni correction $(p=0.0001$; excess of risk for homozygous carriers $=1.85 ; 95 \% \mathrm{CI}=1.25-2.85$; Table 2). This model suggests an advantage for the heterozygous subjects over the homozygous for either allele.

\section{Haplotype analysis}

We determined the LD structure of the genomic region of NTRK3 (Figure 1) within our sample and identified a 47-kb block (block 11 in Figure 1) including the location of rs7180942 and three additional SNPs. We further performed association tests with the HBAT algorithm from the P2BAT software (36), using all possible marker haplotypes contained in that block using heterozygous advantage as inheritance model for the ED phenotype. The stronger association involved a common three-marker haplotype for rs7180942, rs10520676 and rs2162266, respectively $\left(p=7.86 \times 10^{-05}\right.$ in 143 informative families) (Table 3).

\section{Bioinformatics search of functional consequences of rs7180942}

Through the interactive web-based SNP analysis tool Pupasuite (http://pupasuite.bioinfo.cipf.es/) (37) we found that none of the involved SNPs cause 
non synonymous substitutions, nor disrupt putative transcription binding sites, putative exonic splicing enhancers or intron exon boundaries. When the most recent HapMap data (phase II release 22; April 2007) were considered, we estimated that rs7180942 is also capturing the variability of five highly correlated SNPs, namely rs8035239, rs1426300, rs1110306, rs2059588 and rs3825882 (mean R square $=0.95)$. We neither found any putative functional consequences of these SNPs using the Pupa Suite website.

\section{Interaction analysis}

We further looked for a gene-gene effect and performed an interaction analysis to find other variants over or undertransmitted in patients dependent on the rs7180942 genotype. For that purpose, we distinguished between families with a heterozygous descendant for rs7180942 (the undertransmitted genotype in ED patients) and families with a homozygous affected offspring for either allele. Following an additive model, families with homozygous offspring for rs7180942 were found strongly associated with rs17033706, located in NGFB gene, which remained significant after Bonferroni correction. Thus, we observed overtransmission of the A allele in rs17033706 in families with homozygous offspring for rs7180942 (Transmission odds ratio for rs7180942 Homozygous: 13.69 (3.43-54.60), Transmission odds ratio for rs7180942 Heterozygous 3.45 (1.03-11.11), Interaction p value (5.64 x 10 $\left.0^{-5}\right)$ (Table 4).

\section{Effect of rs7180942 genotype in NTRK3 expression}

To asses a possible functional role of this SNP on the expression of NTRK3, we extracted the normalized expression data from HapMap lymphoblastoid cell lines (38) corresponding to Illumina probe GI_4505474.S, located in the 3'coding-UTR boundaries of the full length NTRK3 isoform. When we tested its correlation with the three different genotypes of rs7180942, found that the heterozygous samples presented significantly lower expression levels $(p=0.017)$, which is in agreement with the 
heterozygous advantage / overdominant model of inheritance found to be associated to ED. The other variants within the same 47-kb block (block 11 in Figure 1), rs10520676, rs2162266 and rs3784406, were not associated with the levels of expression of NTRK3.

\section{Ntrk3 expression in the $a n x / a n x$ anorexia mouse model}

The anx anorexia murine model (39) is caused by an autosomal recessive mutation located approximately $20 \mathrm{cM}$ proximal to the agouti locus on mouse chromosome 2. The mutation entails decreased food intake, growth retardation, and emaciated appearance. Because the $a n x / a n x$ mouse is an attractive genetic model of feeding behaviour, we used a comparative functional genomics approach to determine the putative involvement of NTRK3 in the regulation of energy metabolism and feeding. Hence, we determined inter-individual expression differences for Ntrk3 gene in the cortex and hypothalamus of three anx/anx mice and three control littermates by quantitative PCR (TaqMan). We found that the Ntrk3 mRNA levels were increased in the hypothalamus of anx/anx mice, whereas no differences were found in the levels of expression when comparing the cortex $(\mathrm{P}=0.024$, ratio $=1.36$; $95 \% \mathrm{CI}=(1.002-$

1.848); and $\mathrm{P}=0.464$, fold change $=0.98 ; 95 \% \mathrm{CI}=(0.73-1.34)$, respectively) (Figure 2). 


\section{Discussion}

We have carried out a pathway-based candidate gene approach to perform a familybased analysis and tested for association between neurotrophic factors and their receptors and the susceptibility to eating disorders. Both single and multiple-marker analysis showed a strong association between NTRK3 and ED with a heterozygous advantage model of inheritance, which remained significant after correcting for multiple testing. These results are in agreement with the reduced NTRK3 expression levels observed in lymphoblastoid cell lines of heterozygous individuals for rs7180942 (38) and suggest that increased NTRK3 function may contribute to the predisposition to ED. The heterozygous advantage mode of inheritance, also known as molecular heterosis, has received little attention, although it has been postulated as occurring in up to $50 \%$ of all gene associations in psychiatric disorders or personality traits $(40,41)$. Several genes, many of them involved in cognitive and psychiatric problems, such as ADRA2C, DRD1, DRD2, DRD3, DRD4, HTR2A, SLC6A4 have been reported to follow a heterozygous advantage model of inheritance (reviewed in (40)). Examples include the T102C polymorphism of the serotonin 2A receptor gene and neuroticism scores, the dopamine D2 receptor TaqI A1/A2 polimorphism and symptoms of ADHD (42) and pathological gambling (43), or a negative heterosis effect of the DdeI polymorphism in the dopamine D1 receptor gene and the scores for the quantitative traits of alcohol abuse, compulsive eating, gambling and tics (44).

At the biological level, it is well established that all neurotrophin receptors dimerize in response to ligand, leading to activation of the tyrosine kinase domain (reviewed in (45)). At the molecular level, perhaps the function of the NTF3 receptor differs depending on the interaction of the subunit polypeptides and, thus, in the particular case of molecular heterosis, homodimers might be more efficient than receptors with two 
structurally different subunits and, somehow, may participate in the biological susceptibility to AN and BN. Nevertheless, how the particular variants that we have identified, or other functional variants in LD with them, can potentially affect the protein and result in an allosteric effect remains to be clarified.

In fact, we observed nominal associations at all genes for at least one SNP and one ED subtype. Of those, NTRK3 (the high affinity receptor for NTF3) appears to be the gene with more association signals, since eight SNPs were associated to either ED, AN or BN. The strongest association, which was still significant after correcting for multiple testing, was found with the ED phenotype and rs7180942, located in intron 8 of NTRK3. We specifically observed an undertransmission of the heterozygous genotype to the affected offspring, together with an overtransmission of both homozygous genotypes, which is known as heterozygous advantage model of inheritance. The haplotype analysis revealed a significant association between ED and a three-marker composite haplotype, which, in agreement with the single-SNP analysis, also followed a heterozygous advantage model of inheritance. This haplotype extends $47 \mathrm{~kb}$ and points to a region spanning from introns 4 to 8 of $N T R K 3$, and including exons 5 to 9 .

According to Pupa Suite website, we did not find any putative functional consequences of rs7180942 or its highly correlated SNPs according to HapMap. Thus, in the absence of a direct causal implication of our candidate SNPs, the observed association could be in LD with another unraveled functionally important sequence variant within the investigated NTRK3 gene block.

When we tested the potential effect of the rs7180942 variant on the expression of the gene in lymphoblastoid cell lines using the Stranger et al. (2007) data, we found that the heterozygous individuals had lower NTRK3 expression. Thus, in this particular 
case of molecular heterosis, a receptor with two structurally different subunits seems to function less efficiently and somehow protect the individual.

NTF3, the NTRK3 ligand, prevents the death of adult central noradrenergic neurons, promotes survival of ventral mesencephalic dopaminergic neurons, cerebellar granule neurons and Purkinje cells, and acts on sensory or sympathetic neurons of the dorsal root, nodose and sympathetic ganglia (46). Enhanced neurogenesis caused by dietary restriction has been described to be accompanied by increased NTF3 (and BDNF) levels (27). Moreover, increased levels of NTF3 have been described in the cerebrospinal fluid of patients with major depression (47), and decreased NTRK3 levels have been reported in the frontal cortex of patients with schizophrenia (48). To test if NTRK3 has a more specific role in eating behavior, we determined the mRNA levels of the Ntrk3 gene in the hypothalamus and cortex of an anorexia mouse model (anx/anx). Interestingly, although anx/anx mice is just a model of starvation, rather than an eating disorder model, where other psychological aspects are involved, it has been shown to mimic the hypothalamamic deregulation observed in AN patients (49). The anx/anx mice showed increased levels of Ntrk3 only in the hypothalamus, of anx/anx mice, a brain area involved in motivation, satiety, and control of food intake $(50,51)$. Thus, although Ntrk3 does not carry the mutation leading to the anx phenotype nor lies in the region identified to be linked to it (39), it may act as a modulator in the promotion of feeding or satiety.

Transgenic mice overexpressing Ntrk3 do not show body weight nor feeding behavior abnormalities, but display increased anxiety-like behavior (52) and differential responses to anxiogenic drugs (53). Targeted mutation of $N t f 3$ in mice result in a failure to thrive when compared with their wild-type littermates (54), and both $\mathrm{Ntf}^{-/-}$and $\mathrm{Ntrk3}^{-/}$knock-out mice have a reduced number of enteric neurons (55). Since meal 
termination and satiety are mainly vagally mediated functions, it is possible that the role of NTRK3 in human ED is the result of an abnormal autonomic control of the digestive tract that can eventually modulate the information from the peripheral gastrointestinal tract that is conveyed centrally to the central nervous system to modulate feeding behavior.

Additionally, we observed that the risk of ED appears to increase in individuals homozygous for rs7180942 who also carry the A allele of rs17033706 in NGF, while the independent risk contributed by this later SNP to the ED phenotype did not reach statistical significance. This suggests a synergistic epistatic interaction between these genes, in which the phenotypic effect of a putative functional variant in NGF would also depend on the genetic background in NTRK3. Thus, being homozygous carrier at rs7180942 locus in NTRK3 increases the risk of ED and increases the effect of the A rs17033706 allele at NGF on ED. Complex physical and functional interactions between neurotrophins and their receptors are necessary for a homeostatic balance to regulate signal transduction $(56,57)$, and neurotrophin-neurotrophin interactions have also been described in vivo $(58,59)$. For instance, NTF3 is known to bind primary to NTRK3, but has also promiscuous affinity for NTRK1 and NTRK2 (60-62). Moreover, it has been reported that NTF3, through the NGF receptor NTRK1, modulates BDNF levels $(63,64)$. In agreement with that, it has been reported that intracerebroventricular injection of NGF induces hypophagia in rats (26), and it is thus possible that genetic variability in NGF could influence the expression, release, or activity of the protein and affect eating behavior depending on the genetic background of NTRK3 (62).

Our group has previously reported the association of BDNF and NTRK2 in ED (21-25). In the present analysis, we have found positive signals for SNPs in both genes, but since the number of analyzed variants is substantially higher than in the previous 
reports, they did not remain significant after correction for multiple tests. In addition to our investigation $(22,24,25,65)$, the present study also suggests the involvement of NTRK3 and NGF, and put forward the possibility that ED could be the result of an altered cross-regulation of the different members of the neurotrophin signaling pathway. This would entail an unbalanced biochemical interaction between ligands, their receptors and their intracellular target proteins that could eventually affect functions related to both specific central and peripheral neuronal populations. This hypothesis is in agreement with eating behavior being a complex phenomenon which results from the interaction of different brain regions and many factors, including olfactory, visual, emotional and higher cognitive inputs, as well as several nutritional signals coming from the periphery.

Although family-based association studies circumvent the possible false positives due to population admixture and stratification and avoid spurious associations due to differences in allele frequencies among various populations (66), further studies including a larger number of probands are needed in order to conclude which of the nominal associations are true positives and to confirm the interaction between the different molecules. Neurotrophins have been shown to be involved in different mental diseases like major depression, bipolar disorder, schizophrenia or substance abuse (24, 67), but to our knowledge, no interaction between them has been described.

There is substantial comorbidity between ED and psychiatric disorders, and comorbid Axis I and II psychopathology is associated with higher levels of severity in eating disorder symptoms (68-74). It is probable that several factors account for this associations and, although not controlled in our analyses, may represent a manifestation of a shared transmission (Keel, Klump, Miller, McGue, 2005). Thus, we propose that this study presents an interesting unexplored genomic pathway to screen that could 
validate the neurotrophin hypothesis role on the development of psychiatric disorders $(19,67)$. 


\section{Materials and Methods}

\section{Subjects and phenotype}

All subjects fulfilled DSM-IV (Diagnostic and Statistical Manual of Mental Disorders, 4th edition) criteria for ED. All the participants were diagnosed as having ED as the main disorder with the Structured Clinical Interview for Mental Disorders, research version 2.0 (SCID-I) for the Spanish patients, the Diagnostic Interview for Genetic Studies (DIGS) for the French patients, and the Composite International Diagnostic Interview (CIDI) for the German participants. All the patients were women and most of them have been studied in previous reports $(21,75,76)$. Diagnosis was blind to genotype. The study had ethics approval from each center ethics committee and written informed consent was obtained from all participants.

After excluding 15 cases due to false paternity, the dataset consisted of 371ED patients: 121 from Spain, 168 from France and 82 from Germany. Clinical subtypes of each population are summarized in Table 1. Briefly, we obtained 286 AN trios: 142 restricting subtype (ANR), 127 purging subtype (ANP) and 17 not yet classified as restricting or binge-eating/purging subtype due to insufficient time elapsed (3 years) of restricting illness. Finally, we also obtained 59 BN trios: 55 binge-eating/purging (BNP), 2 non-purging subtype (BNNP) and 2 cases not classified as bingeeating/purging or non-purging subtype, and 26 ED not otherwise specified (EDNOS). The lifetime minimum body mass index (minBMI) was $14.0 \mathrm{~kg} / \mathrm{m}^{2}(13.7-14.24)$ for AN patients and $19.0 \mathrm{~kg} / \mathrm{m}^{2}$ (18.2-19.85) for BN patients. The lifetime maximum body mass index (maxBMI) was $21.2 \mathrm{~kg} / \mathrm{m}^{2}$ (20.7-21.7) for AN patients and $25.9 \mathrm{~kg} / \mathrm{m}^{2}$ (24.427.3) for BN patients. The average age at onset was 16.5 (15.9-16.99) for AN patients and 18.5 (17-19.99) for BN patients.

\section{SNPs and Genotyping}


From the HapMap project data set, we utilized genotypes from the public release 16 (phase I data freeze; dbSNP b124) corresponding to the 90 individuals from the CEPH 30 trios of European descent (http://www.hapmap.org). Only SNPs having a unique mapping location on the NCBI B34 assembly and a minor allele frequency (MAF) higher than 10\% were considered for further analysis. Bins of common SNPs in strong LD, as defined by r2 higher than 0.85 , were identified within this data set by using HapMap-LDSelect-Processor, which uses the 'LD Select’ method to process HapMap genotype dump format data corresponding to the locus region for each gene, covering the entire gene and $10 \mathrm{~kb}$ upstream and downstream the gene. 183 TagSNPs were selected for coverage of all bins for a total of 10 genes (Supplementary Table 1). Block structure according to our data was evaluated by Haploview software version 3.2 (77), using the Solid Spine of $L D$ method.

TagSNPs were genotyped using the SNPlex Genotyping System. SNPs that passed the design rules for the development of good assays were selected. For the genotyping process, we followed the manufacturer's protocol for the SNPlex Multiplex Genotyping Systems (Applied Biosystems, Foster City, CA, USA): $12 \mu$ of each DNA sample at $75 \mathrm{ng} / \mu \mathrm{l}$ were fragmented by boiling at $99^{\circ} \mathrm{C}$ for $10 \mathrm{~min}$ and $2 \mu \mathrm{l}(150 \mathrm{ng})$ were allowed to dehydrate in a 384-well plate. Four custom multiplex pools (currently up to 48-plex) were designed, synthesized and formulated by the manufacturer. The manufacturer's specifications were followed through phosphorylation, oligonucleotide ligation, exonuclease clean-up, PCR and hybridization steps (Applied Biosystems, Foster City, CA, USA). All reactions were set up in a 384-well format using various robotic systems. The high-throughput genotyping assays were performed at the genotyping facilities of CeGen, in the Barcelona Node (Centro Nacional de Genotipado, Genoma España). 
Researchers performing the genotypes were blinded respect to phenotype. In addition to internal positive and negative controls provided by ABI for the SNPlex procedure, we analyzed the genotype concordance and the Mendelian inheritance in six samples corresponding to two HapMap reference trios: samples NA10860, NA10861, NA11992, NA11993, NA11994 and NA11995 (family numbers CEPH131 and CEPH132). Genotype concordance was tested using SNPator (http://www.CEGEN.org) and Mendelian inheritance was tested using FBAT software. Families with more than three SNPs that presented Mendelian errors were excluded from the analysis. HardyWeinberg equilibrium and LD of SNPs were assessed by the Haploview v2.03 software.

\section{Sample power calculation}

The power of our sample was calculated with the HelixTree software (Golden Helix, Bozeman, MT, USA), assuming a population prevalence of $2 \%$, an allele frequency of 0.1 and Odds Ratio of 1.5 . The minimum power of the whole sample was $77 \%$. For the haplotype analysis, the power was calculated post-hoc with HelixTree software. Finally, the power to detect an interaction effect of 1.8 in a sample of 350 trios was $98 \%$ and was calculated post hoc with the Quanto software $(78,79)$.

\section{Heterogeneity analysis}

We excluded genetic heterogeneity among centers comparing allelic frequencies of the patients' parents from the different centers with the SNPassoc software (80), that carries out likelihood ratio statistic tests, for all SNPs.

\section{Family-based association study}

For the family-based association study we used FBAT (81), and P2BAT (36), which is implemented in HelixTree version 5.2.0 (Golden Helix, Inc, Bozeman, MT). Using the FBAT software, the codominant model that computes Z scores for the three possible genotypes, was used as a first screening for the single-marker analysis. Bonferroni 
correction for 150 SNP that passed genotyping quality controls and ED subtypes (total $\mathrm{ED}, \mathrm{AN}$ and $\mathrm{BN}$ ), was used to for multiple comparisons. Thus, significant p values were lowered to $\mathrm{p}=1.11 \times 10^{-4}$.

Haplotype analysis was performed with the HBAT algorithm from the P2BAT software (36) implemented in HelixTree (Golden Helix, Bozeman, MT, USA), which computes haplotypes before executing FBAT using bi-allelic and multi-allelic modes, and which permits the use of the heterozygous advantage/overdominant model. The Jiang et al approach (82) was used for the Family-Based Association analysis.

Bonferroni correction was applied taking into account the number of tests calculated by the P2BAT software.

\section{Interaction Analysis}

We performed conditional logistic regressions to find out interactions with rs7180942 and all the other SNPs, except those ones in NTRK3. For this purpose we identified the families with homozygous offspring for both rs7180942 alleles and with offspring heterozygous for rs7180942. The conditional logistic regression models will take into account the matched pair of observations generated by each parent-proband. For each analysis we calculated the odds of transmission for the over transmitted allele $\left(A_{i}\right)$ of the SNP of interest vs. its non-transmission for both homozygous and heterozygous offspring's for rs7180942. The logistic regression model is:

$\log \left[\frac{P(\text { transmission })}{1-P(\text { transmission })}\right]=\beta_{0}+\beta_{1} A_{i}+\beta_{2} r s 7180942+\gamma A_{i} * r s 7180942$

where testing the hypothesis that $\gamma=0$ becomes the test of gene-gene interaction. We calculated the log likelihood ratio statistic for hypothesis testing. Furthermore, $e^{\beta_{1}}$ represents the odds ratio for transmission of $A_{i}$ to the heterozygous for rs7180942 
and $e^{\beta_{1}+\gamma}$ is the odds ratio for transmission of $\mathrm{A}_{\mathrm{i}}$ to the homozygous offsprings for rs718042.

To ensure a global type I error of at most 0.05 , we used the full interaction strategy for Bonferroni correction (83) and set the significance level of the test to $4.85 \times 10^{-4}$.

\section{Association of NTRK3 SNPs with expression levels}

Whole genome Illumina lymphoblastoid cell line gene expression data from 210 unrelated HapMap individuals was extracted from the GSE6536 series dataset in Gene Expression Omnibus (GEO) site (http://www.ncbi.nlm.nih.gov/geo) (38). The probe of interest, in that case probe GI_4505474.S, located in the 3'coding / 3’UTR boundaries of NTRK3 gene, was selected for the analysis. Genotype data of rs7180942 from 210 unrelated individuals from CEPH (from European, African and Asian ancestry) was downloaded from HapMap browser (http://www.hapmap.org/cgiperl/gbrowse/hapmap_B35/). The association analysis between this SNP and the levels of expression for probe GI_4505474.S was tested using SNP assoc software for the different genetic models, as described above.

\section{Quantification of the levels of expression of Ntrk3 in the anx/anx murine model}

Briefly, three anx/anx (B6C3Fe a/a- anx/J) and three wild type (heterozygous or homozygous wild-type) littermates were anesthetized with isofluoran and sacrificed at postnatal day 14. Hypothalamus and cortex were dissected and frozen on liquid nitrogen. RNA was extracted through standard methods using Trizol ${ }^{\mathrm{TM}}$. cDNA was obtained from total RNA using the High Capacity Archive Kit from Applied Biosystems (Applied Biosystems, Foster City, CA, USA). Pre-designed Taqman assays (Applied Biosystems, Foster City, CA, USA) were used to analyze the relative quantitative expression of Ntrk3 and three endogenous controls were used to normalize the expression levels. The probes used were Mm00456222_m1 for Ntrk3, and 
endogenous controls were Mm00495501_m1 for Xpr1, Mm00446973_m1 for Tbp and Mm01158417_g1 for Ywhaz. Two replicates were performed for each real-time PCR experiments. Relative quantities were calculated using qbase software (84) and the geometric mean of the three endogenous controls was used to normalize the expression. Relative quantities were calculated using qbase software (78) and the geometric mean of the three endogenous controls was used to normalize the expression. A linear mixed model (85) (LMM) was used to compare the wild type versus anx/anx expression. The LMM included the genotype (wild type and anx/anx), the tissue (hypothalamus and cortex) and their interaction as fixed effects. The littermates were included in the model as random effects in order to control the dependency of the 4 measures taken from each littermate. 


\section{Acknowledgements}

Financial support was received from the Ministry of Education and Science (SAF200501005), the "Fondo de Investigaciones Sanitarias de la Seguridad Social, FIS (PI040632; PI040619), the Department of Health (Generalitat de Catalunya), and the Department of Universities, Research and Information Society (2005SGR00008; 2005SGR 00322) (Generalitat de Catalunya), “EC Framework V 'Factors in Healthy Eating’ consortium (QLK1-1999-916) and Bundesministerium für Bildung und Forschung (NGFN2 01GS0482, 01GS0483), and the European Union (FP6 LSHMCT2003-503041). Josep M. Mercader was supported by the CRG under project SAF200200799 (Spanish Ministry of Science and Education), and by fellowship of the Danone Institute. We thank also the support from Genome Spain to the National Genotyping Center (CeGen). We finally thank Anna Wald for critically reading the manuscript.

\section{Conflict of Interest Statement}

The authors declare that they do not have conflicts of interest 


\section{References:}

1. A.P.A. (1994) DSM-IV: Diagnostic and Statistical Manual of Mental Disorders. The American Psychiatric Association, Washington, D. C.

2. Keski-Rahkonen, A., Hoek, H.W., Susser, E.S., Linna, M.S., Sihvola, E., Raevuori, A., Bulik, C.M., Kaprio, J. and Rissanen, A. (2007) Epidemiology and course of anorexia nervosa in the community. Am J Psychiatry, 164, 1259-1265.

3. Garner, D.M. (1993) Pathogenesis of anorexia nervosa. Lancet, 341, 1631-1635.

4. Treasure, J. and Campbell, I. (1994) The case for biology in the aetiology of anorexia nervosa. Psychol Med, 24, 3-8.

5. $\quad$ Klump, K.L., Miller, K.B., Keel, P.K., McGue, M. and Iacono, W.G. (2001) Genetic and environmental influences on anorexia nervosa syndromes in a population-based twin sample. Psychol Med, 31, 737-740.

6. Wade, T.D., Bulik, C.M., Neale, M. and Kendler, K.S. (2000) Anorexia nervosa and major depression: shared genetic and environmental risk factors. Am $J$ Psychiatry, 157, 469-471.

7. Kendler, K.S., MacLean, C., Neale, M., Kessler, R., Heath, A. and Eaves, L. (1991) The genetic epidemiology of bulimia nervosa. Am J Psychiatry, 148, 1627-1637.

8. Sullivan, P.F., Bulik, C.M. and Kendler, K.S. (1998) Genetic epidemiology of binging and vomiting. Br J Psychiatry, 173, 75-79.

9. Bulik, C.M., Sullivan, P.F. and Kendler, K.S. (1998) Heritability of binge-eating and broadly defined bulimia nervosa. Biol Psychiatry, 44, 1210-1218.

10. Walters, E.E. and Kendler, K.S. (1995) Anorexia nervosa and anorexic-like syndromes in a population-based female twin sample. Am J Psychiatry, 152, 6471.

11. Lilenfeld, L.R., Kaye, W.H., Greeno, C.G., Merikangas, K.R., Plotnicov, K., Pollice, C., Rao, R., Strober, M., Bulik, C.M. and Nagy, L. (1998) A controlled family study of anorexia nervosa and bulimia nervosa: psychiatric disorders in first-degree relatives and effects of proband comorbidity. Arch Gen Psychiatry, 55, 603-610.

12. Strober, M., Freeman, R., Lampert, C., Diamond, J. and Kaye, W. (2000) Controlled family study of anorexia nervosa and bulimia nervosa: evidence of shared liability and transmission of partial syndromes. Am J Psychiatry, 157, 393-401.

13. Schor, N.F. (2005) The p75 neurotrophin receptor in human development and disease. Prog Neurobiol, 77, 201-214.

14. Lu, B., Pang, P.T. and Woo, N.H. (2005) The yin and yang of neurotrophin action. Nat Rev Neurosci, 6, 603-614.

15. Schinder, A.F. and Poo, M. (2000) The neurotrophin hypothesis for synaptic plasticity. Trends Neurosci, 23, 639-645.

16. Kernie, S.G., Liebl, D.J. and Parada, L.F. (2000) BDNF regulates eating behavior and locomotor activity in mice. Embo J, 19, 1290-1300.

17. Bersani, G., Iannitelli, A., Fiore, M., Angelucci, F. and Aloe, L. (2000) Data and hypotheses on the role of nerve growth factor and other neurotrophins in psychiatric disorders. Med Hypotheses, 55, 199-207.

18. Duman, R.S., Heninger, G.R. and Nestler, E.J. (1997) A molecular and cellular theory of depression. Arch Gen Psychiatry, 54, 597-606. 
19. Lang, U.E., Jockers-Scherubl, M.C. and Hellweg, R. (2004) State of the art of the neurotrophin hypothesis in psychiatric disorders: implications and limitations. J Neural Transm, 111, 387-411.

20. Xu, B., EH., G., Zang, K., Cepoi, D., Cone, R.D., Jones, R.K., Tecott, L.H. and Reichardt, L.F. (2003) Brain-derived neurotrophic factor regulates energy balance downstream of melanocortin-4 receptor. Nature Neuroscience, 6, 736742.

21. Ribases, M., Gratacos, M., Fernandez-Aranda, F., Bellodi, L., Boni, C., Anderluh, M., Cristina Cavallini, M., Cellini, E., Di Bella, D., Erzegovesi, S. et al. (2005) Association of BDNF with restricting anorexia nervosa and minimum body mass index: a family-based association study of eight European populations. Eur J Hum Genet, 13, 428-434.

22. Ribases, M., Gratacos, M., Badia, A., Jimenez, L., Solano, R., Vallejo, J., Fernandez-Aranda, F. and Estivill, X. (2005) Contribution of NTRK2 to the genetic susceptibility to anorexia nervosa, harm avoidance and minimum body mass index. Mol Psychiatry, 10, 851-860.

23. Ribases, M., Gratacos, M., Armengol, L., de Cid, R., Badia, A., Jimenez, L., Solano, R., Vallejo, J., Fernandez, F. and Estivill, X. (2003) Met66 in the brainderived neurotrophic factor (BDNF) precursor is associated with anorexia nervosa restrictive type. Mol Psychiatry, 8, 745-751.

24. Gratacos, M., Gonzalez, J.R., Mercader, J.M., de Cid, R., Urretavizcaya, M. and Estivill, X. (2007) Brain-derived neurotrophic factor Val66Met and psychiatric disorders: meta-analysis of case-control studies confirm association to substance-related disorders, eating disorders, and schizophrenia. Biol Psychiatry, 61, 911-922.

25. Mercader, J.M., Ribases, M., Gratacos, M., Gonzalez, J.R., Bayes, M., de Cid, R., Badia, A., Fernandez-Aranda, F. and Estivill, X. (2007) Altered brainderived neurotrophic factor blood levels and gene variability are associated with anorexia and bulimia. Genes Brain Behav, 6, 706-716.

26. Williams, L.R. (1991) Hypophagia is induced by intracerebroventricular administration of nerve growth factor. Exp Neurol, 113, 31-37.

27. Lee, J., Seroogy, K.B. and Mattson, M.P. (2002) Dietary restriction enhances neurotrophin expression and neurogenesis in the hippocampus of adult mice. $J$ Neurochem, 80, 539-547.

28. Chi, M.M., Fan, G. and Fox, E.A. (2004) Increased short-term food satiation and sensitivity to cholecystokinin in neurotrophin-4 knock-in mice. Am J Physiol Regul Integr Comp Physiol, 287, R1044-1053.

29. Byerly, M.S. and Fox, E.A. (2006) High-fat hyperphagia in neurotrophin-4 deficient mice reveals potential role of vagal intestinal sensory innervation in long-term controls of food intake. Neurosci Lett, 400, 240-245.

30. Emerich, D.F., Winn, S.R., Hantraye, P.M., Peschanski, M., Chen, E.Y., Chu, Y., McDermott, P., Baetge, E.E. and Kordower, J.H. (1997) Protective effect of encapsulated cells producing neurotrophic factor CNTF in a monkey model of Huntington's disease. Nature, 386, 395-399.

31. Sleeman, M.W., Garcia, K., Liu, R., Murray, J.D., Malinova, L., Moncrieffe, M., Yancopoulos, G.D. and Wiegand, S.J. (2003) Ciliary neurotrophic factor improves diabetic parameters and hepatic steatosis and increases basal metabolic rate in db/db mice. Proc Natl Acad Sci U S A, 100, 14297-14302. 
32. Kokoeva, M.V., Yin, H. and Flier, J.S. (2005) Neurogenesis in the hypothalamus of adult mice: potential role in energy balance. Science, 310, 679683.

33. Xu, B., Dube, M.G., Kalra, P.S., Farmerie, W.G., Kaibara, A., Moldawer, L.L., Martin, D. and Kalra, S.P. (1998) Anorectic effects of the cytokine, ciliary neurotropic factor, are mediated by hypothalamic neuropeptide Y: comparison with leptin. Endocrinology, 139, 466-473.

34. Lambert, P.D., Anderson, K.D., Sleeman, M.W., Wong, V., Tan, J., Hijarunguru, A., Corcoran, T.L., Murray, J.D., Thabet, K.E., Yancopoulos, G.D. et al. (2001) Ciliary neurotrophic factor activates leptin-like pathways and reduces body fat, without cachexia or rebound weight gain, even in leptinresistant obesity. Proc Natl Acad Sci U S A, 98, 4652-4657.

35. Martin, D., Merkel, E., Tucker, K.K., McManaman, J.L., Albert, D., Relton, J. and Russell, D.A. (1996) Cachectic effect of ciliary neurotrophic factor on innervated skeletal muscle. Am J Physiol, 271, R1422-1428.

36. Hoffmann, T. and Lange, C. (2006) P2BAT: a massive parallel implementation of PBAT for genome-wide association studies in R. Bioinformatics, 22, 31033105.

37. Conde, L., Vaquerizas, J.M., Dopazo, H., Arbiza, L., Reumers, J., Rousseau, F., Schymkowitz, J. and Dopazo, J. (2006) PupaSuite: finding functional single nucleotide polymorphisms for large-scale genotyping purposes. Nucleic Acids Res, 34, W621-625.

38. Stranger, B.E., Forrest, M.S., Dunning, M., Ingle, C.E., Beazley, C., Thorne, N., Redon, R., Bird, C.P., de Grassi, A., Lee, C. et al. (2007) Relative impact of nucleotide and copy number variation on gene expression phenotypes. Science, 315, 848-853.

39. Maltais, L.J., Lane, P.W. and Beamer, W.G. (1984) Anorexia, a recessive mutation causing starvation in preweanling mice. J Hered, 75, 468-472.

40. Comings, D.E. and MacMurray, J.P. (2000) Molecular heterosis: a review. Mol Genet Metab, 71, 19-31.

41. Comings, D.E. (1999) Molecular heterosis as the explanation for the controversy about the effect of the DRD2 gene on dopamine D2 receptor density. Mol Psychiatry, 4, 213-215.

42. Keel, P.K., Klump, K.L., Miller, K.B., McGue, M. and Iacono, W.G. (2005) Shared transmission of eating disorders and anxiety disorders. Int J Eat Disord, 38, 99-105.

43. Comings, D.E., Rosenthal, R.J., Lesieur, H.R., Rugle, L.J., Muhleman, D., Chiu, C., Dietz, G. and Gade, R. (1996) A study of the dopamine D2 receptor gene in pathological gambling. Pharmacogenetics, 6, 223-234.

44. Comings, D.E., Gade, R., Wu, S., Chiu, C., Dietz, G., Muhleman, D., Saucier, G., Ferry, L., Rosenthal, R.J., Lesieur, H.R. et al. (1997) Studies of the potential role of the dopamine D1 receptor gene in addictive behaviors. Mol Psychiatry, 2, 44-56.

45. Schramm, A., Schulte, J.H., Astrahantseff, K., Apostolov, O., Limpt, V., Sieverts, H., Kuhfittig-Kulle, S., Pfeiffer, P., Versteeg, R. and Eggert, A. (2005) Biological effects of TrkA and TrkB receptor signaling in neuroblastoma. Cancer Lett, 228, 143-153.

46. Ernfors, P. (2001) Local and target-derived actions of neurotrophins during peripheral nervous system development. Cell Mol Life Sci, 58, 1036-1044. 
47. Hock, C., Heese, K., Muller-Spahn, F., Huber, P., Riesen, W., Nitsch, R.M. and Otten, U. (2000) Increased cerebrospinal fluid levels of neurotrophin 3 (NT-3) in elderly patients with major depression. Mol Psychiatry, 5, 510-513.

48. Schramm, M., Falkai, P., Feldmann, N., Knable, M.B. and Bayer, T.A. (1998) Reduced tyrosine kinase receptor $\mathrm{C}$ mRNA levels in the frontal cortex of patients with schizophrenia. Neurosci Lett, 257, 65-68.

49. Johansen, J.E., Fetissov, S.O., Bergstrom, U., Nilsson, I., Fay, C., Ranscht, B., Hokfelt, T. and Schalling, M. (2007) Evidence for hypothalamic dysregulation in mouse models of anorexia as well as in humans. Physiol Behav, 92, 278-282.

50. Coll, A.P., Farooqi, I.S. and O'Rahilly, S. (2007) The hormonal control of food intake. Cell, 129, 251-262.

51. Stellar, E. (1994) The physiology of motivation. 1954. Psychol Rev, 101, 301311.

52. Dierssen, M., Gratacos, M., Sahun, I., Martin, M., Gallego, X., Amador-Arjona, A., Martinez de Lagran, M., Murtra, P., Marti, E., Pujana, M.A. et al. (2006) Transgenic mice overexpressing the full-length neurotrophin receptor TrkC exhibit increased catecholaminergic neuron density in specific brain areas and increased anxiety-like behavior and panic reaction. Neurobiol Dis, 24, 403-418.

53. Sahun, I., Delgado-Garcia, J.M., Amador-Arjona, A., Giralt, A., Alberch, J., Dierssen, M. and Gruart, A. (2007) Dissociation between CA3-CA1 synaptic plasticity and associative learning in TgNTRK3 transgenic mice. J Neurosci, 27, 2253-2260.

54. Tessarollo, L., Vogel, K.S., Palko, M.E., Reid, S.W. and Parada, L.F. (1994) Targeted mutation in the neurotrophin-3 gene results in loss of muscle sensory neurons. Proc Natl Acad Sci U S A, 91, 11844-11848.

55. Chalazonitis, A., Pham, T.D., Rothman, T.P., DiStefano, P.S., Bothwell, M., Blair-Flynn, J., Tessarollo, L. and Gershon, M.D. (2001) Neurotrophin-3 is required for the survival-differentiation of subsets of developing enteric neurons. J Neurosci, 21, 5620-5636.

56. Ivanisevic, L., Banerjee, K. and Saragovi, H.U. (2003) Differential crossregulation of TrkA and TrkC tyrosine kinase receptors with p75. Oncogene, 22, 5677-5685.

57. Ivanisevic, L., Zheng, W., Woo, S.B., Neet, K.E. and Saragovi, H.U. (2007) TrkA receptor "hot spots" for binding of NT-3 as a heterologous ligand. J Biol Chem, 282, 16754-16763.

58. Lindholm, D., da Penha Berzaghi, M., Cooper, J., Thoenen, H. and Castren, E. (1994) Brain-derived neurotrophic factor and neurotrophin-4 increase neurotrophin-3 expression in the rat hippocampus. Int J Dev Neurosci, 12, 745751.

59. Karchewski, L.A., Gratto, K.A., Wetmore, C. and Verge, V.M. (2002) Dynamic patterns of BDNF expression in injured sensory neurons: differential modulation by NGF and NT-3. Eur J Neurosci, 16, 1449-1462.

60. Cordon-Cardo, C., Tapley, P., Jing, S.Q., Nanduri, V., O'Rourke, E., Lamballe, F., Kovary, K., Klein, R., Jones, K.R., Reichardt, L.F. et al. (1991) The trk tyrosine protein kinase mediates the mitogenic properties of nerve growth factor and neurotrophin-3. Cell, 66, 173-183.

61. Squinto, S.P., Stitt, T.N., Aldrich, T.H., Davis, S., Bianco, S.M., Radziejewski, C., Glass, D.J., Masiakowski, P., Furth, M.E., Valenzuela, D.M. et al. (1991) trkB encodes a functional receptor for brain-derived neurotrophic factor and neurotrophin-3 but not nerve growth factor. Cell, 65, 885-893. 
62. Huang, E.J. and Reichardt, L.F. (2001) Neurotrophins: roles in neuronal development and function. Annu Rev Neurosci, 24, 677-736.

63. Xu, B., Michalski, B., Racine, R.J. and Fahnestock, M. (2002) Continuous infusion of neurotrophin-3 triggers sprouting, decreases the levels of TrkA and TrkC, and inhibits epileptogenesis and activity-dependent axonal growth in adult rats. Neuroscience, 115, 1295-1308.

64. Ullal, G.R., Michalski, B., Xu, B., Racine, R.J. and Fahnestock, M. (2007) NT-3 modulates BDNF and proBDNF levels in naive and kindled rat hippocampus. Neurochem Int, 50, 866-871.

65. Ribases, M., Gratacos, M., Fernandez-Aranda, F., Bellodi, L., Boni, C., Anderluh, M., Cavallini, M.C., Cellini, E., Di Bella, D., Erzegovesi, S. et al. (2004) Association of BDNF with anorexia, bulimia and age of onset of weight loss in six European populations. Hum Mol Genet, 13, 1205-1212.

66. Laird, N.M. and Lange, C. (2006) Family-based designs in the age of large-scale gene-association studies. Nat Rev Genet, 7, 385-394.

67. Chao, M.V., Rajagopal, R. and Lee, F.S. (2006) Neurotrophin signalling in health and disease. Clin Sci (Lond), 110, 167-173.

68. Fernandez-Aranda, F., Jimenez-Murcia, S., Alvarez-Moya, E.M., Granero, R., Vallejo, J. and Bulik, C.M. (2006) Impulse control disorders in eating disorders: clinical and therapeutic implications. Compr Psychiatry, 47, 482-488.

69. Fernandez-Aranda, F., Pinheiro, A.P., Tozzi, F., Thornton, L.M., Fichter, M.M., Halmi, K.A., Kaplan, A.S., Klump, K.L., Strober, M., Woodside, D.B. et al. (2007) Symptom profile of major depressive disorder in women with eating disorders. Aust N Z J Psychiatry, 41, 24-31.

70. Godart, N.T., Flament, M.F., Lecrubier, Y. and Jeammet, P. (2000) Anxiety disorders in anorexia nervosa and bulimia nervosa: co-morbidity and chronology of appearance. Eur Psychiatry, 15, 38-45.

71. Grilo, C.M., Sanislow, C.A., Skodol, A.E., Gunderson, J.G., Stout, R.L., Shea, M.T., Zanarini, M.C., Bender, D.S., Morey, L.C., Dyck, I.R. et al. (2003) Do eating disorders co-occur with personality disorders? Comparison groups matter. Int J Eat Disord, 33, 155-164.

72. $\quad$ Kaye, W.H., Bulik, C.M., Thornton, L., Barbarich, N. and Masters, K. (2004) Comorbidity of anxiety disorders with anorexia and bulimia nervosa. Am J Psychiatry, 161, 2215-2221.

73. Milos, G., Spindler, A. and Schnyder, U. (2004) Psychiatric comorbidity and Eating Disorder Inventory (EDI) profiles in eating disorder patients. Can $J$ Psychiatry, 49, 179-184.

74. $\quad$ Steiger, H., Gauvin, L., Engelberg, M.J., Ying Kin, N.M., Israel, M., Wonderlich, S.A. and Richardson, J. (2005) Mood- and restraint-based antecedents to binge episodes in bulimia nervosa: possible influences of the serotonin system. Psychol Med, 35, 1553-1562.

75. Gorwood, P., Ades, J., Bellodi, L., Cellini, E., Collier, D.A., Di Bella, D., Di Bernardo, M., Estivill, X., Fernandez-Aranda, F., Gratacos, M. et al. (2002) The 5-HT(2A) -1438G/A polymorphism in anorexia nervosa: a combined analysis of 316 trios from six European centres. Mol Psychiatry, 7, 90-94.

76. Gabrovsek, M., Brecelj-Anderluh, M., Bellodi, L., Cellini, E., Di Bella, D., Estivill, X., Fernandez-Aranda, F., Freeman, B., Geller, F., Gratacos, M. et al. (2004) Combined family trio and case-control analysis of the COMT Val158Met polymorphism in European patients with anorexia nervosa. Am J Med Genet, 124B, 68-72. 
77. Barrett, J.C., Fry, B., Maller, J. and Daly, M.J. (2005) Haploview: analysis and visualization of LD and haplotype maps. Bioinformatics, 21, 263-265.

78. Gauderman, W.J. (2002) Sample size requirements for association studies of gene-gene interaction. Am J Epidemiol, 155, 478-484.

79. Gauderman, W.J. and Morrison, J.M. (2006) QUANTO 1.1: A computer program for power and sample size calculations for genetic-epidemiology studies. http://hydra.usc.edu/gxe.

80. Gonzalez, J.R., Armengol, L., Sole, X., Guino, E., Mercader, J.M., Estivill, X. and Moreno, V. (2007) SNPassoc: an R package to perform whole genome association studies. Bioinformatics, 23, 644-645.

81. Horvath, S., Laird, N.M. and Knapp, M. (2000) The transmission/disequilibrium test and parental-genotype reconstruction for X-chromosomal markers. Am J Hum Genet, 66, 1161-1167.

82. Jiang, H., Harrington, D., Raby, B.A., Bertram, L., Blacker, D., Weiss, S.T. and Lange, C. (2006) Family-based association test for time-to-onset data with timedependent differences between the hazard functions. Genet Epidemiol, 30, 124132.

83. Marchini, J., Donnelly, P. and Cardon, L.R. (2005) Genome-wide strategies for detecting multiple loci that influence complex diseases. Nat Genet, 37, 413-417.

84. Hellemans, J., Mortier, G., De Paepe, A., Speleman, F. and Vandesompele, J. (2007) qBase relative quantification framework and software for management and automated analysis of real-time quantitative PCR data. Genome Biol, 8, R19.

85. McCulloch, C.E. and Searle, S.R. (2001) Generalized, Linear, and Mixed Models. Canada: Wiley Series in Probability and Statistics. 


\section{Figure legends}

Figure 1. FBAT results and P values for the NTRK3 SNPs analyzed in the present study with ED, AN and BN phenotypes. Bars are only represented when $\mathrm{P}<0.05$. The bottom line represents the threshold for nominal P values (0.05), the upper line represents thee threshold for Bonferroni correction. NTRK3 isoforms are represented as well as the location of the Illumina probe GI_4505474-S probe used in the analysis of the effect of the rs7180942 genotype in NTRK3 expression. Below, LD plot of the region. Colors represent D’ values and numbers the corresponding R square values.

Figure 2. Box plot representing normalized gene expression values of Ntrk3, with depending on the genotypes. Anx/anx : homozygous mutant mice; anx/?: heterozygous or homozygous wild type mouse; cx: cortex; ht: hypothalamus. 
Table 1. Distribution of clinical subtypes of patients with eating disorders in the different populations and demographical information

\begin{tabular}{|c|c|c|c|c|}
\hline Phenotype & Spain Trios & France Trios & German Trios & Total \\
\hline Eating Disorders & 121 & 168 & 82 & 371 \\
\hline Anorexia Nervosa & 54 & 168 & 64 & 286 \\
\hline Restrictive AN & 23 & 82 & 37 & 142 \\
\hline Purging AN & 31 & 83 & 13 & 127 \\
\hline Bulimia Nervosa & 53 & 0 & 6 & 59 \\
\hline Purgative BN & 51 & 0 & 4 & 55 \\
\hline Non-purging BN & 2 & 0 & 0 & 2 \\
\hline EDNOS & 14 & 0 & 12 & 26 \\
\hline AN MinBMI $(95 \% C I)$ & $15.4(15-15.9)$ & 13.7 (13.4-13.9) & $13.8(13.2-14.4)$ & $14(13.7-14.2)$ \\
\hline AN Age at onset $(95 \% \mathrm{CI})$ & $18(17-19.0)$ & $16.1(15.5-16.7)$ & $15.4(14.7-16.1)^{*}$ & 16.5 (15.9-16.9) \\
\hline BN MinBMI (95\%CI) & 19.1(18.3-19.9) & NA & NA & $19(18.2-19.8)$ \\
\hline BN Age at onset $(95 \% \mathrm{CI})$ & 18.5(17-19.99) & NA & NA & 18.5(17-19.99) \\
\hline
\end{tabular}

NA, non-available information; *In the case of the Marburg/Essen sample, age at onset of weight loss was assessed instead of age at onset; ED, eating disorders; AN, anorexia Nervosa; BN, Bulimia Nervosa; EDNOS, ED not otherwise specified; MinBMI, minimum body mass index. 
Table 2. Association of NTRK3 rs7180942 in eating disorder phenotypes

Eating Disorders

\begin{tabular}{cccccccc}
\hline Genotype & Frequency & Fam\# & S & E(S) & Var(S) & Z & P \\
\hline C/C & 0.231 & 110 & 57 & 43 & 24.5 & 2.828 & $0.005^{*}$ \\
C/T & 0.472 & 166 & 58 & 83 & 41.5 & -3.881 & $1.0 \times 10^{-4} * *$ \\
T/T & 0.296 & 108 & 53 & 42 & 24 & 2.245 & $0.025^{*}$
\end{tabular}

Anorexia Nervosa

$\begin{array}{cccccccc}\text { Genotype } & \text { Frequency } & \text { Fam\# } & \text { S } & \text { E(S) } & \text { Var(S) } & \text { Z } & \text { P } \\ \text { C/C } & 0.231 & 77 & 40 & 29.5 & 17 & 2.547 & 0.011^{*} \\ \mathrm{C} / \mathrm{T} & 0.472 & 119 & 42 & 59.5 & 29.75 & -3.208 & 0.001^{*} \\ \mathrm{~T} / \mathrm{T} & 0.296 & 78 & 37 & 30 & 17.25 & 1.685 & 0.092\end{array}$

Anorexia Nervosa Purging

\begin{tabular}{ccccccccc}
\hline Genotype & Frequency & Fam\# & S & E(S) & Var(S) & Z & P \\
\hline C/C & 0.231 & 32 & 19 & 12.75 & 7.188 & 2.331 & $0.02^{*}$ \\
C/T & 0.472 & 42 & 12 & 21 & 10.5 & -2.777 & $0.005^{*}$ \\
T/T & 0.296 & 23 & 11 & 8.25 & 4.938 & 1.238 & 0.216
\end{tabular}

Anorexia Nervosa Restrictive

\begin{tabular}{ccccccccc}
\hline Genotype & Frequency & Fam\# & S & E(S) & Var(S) & Z & P \\
\hline C/C & 0.231 & 40 & 21 & 15.25 & 8.812 & 1.937 & 0.053
\end{tabular}




$\begin{array}{llllcccc}\mathrm{C} / \mathrm{T} & 0.472 & 67 & 25 & 33.5 & 16.75 & -2.077 & 0.038^{*} \\ \mathrm{~T} / \mathrm{T} & 0.296 & 46 & 21 & 18.25 & 10.312 & 0.856 & 0.392\end{array}$

Bulimia Nervosa Purging

\begin{tabular}{cccccccc}
\hline Genotype & Frequency & Fam\# & S & E(S) & Var(S) & $\mathbf{Z}$ & $\mathbf{P}$ \\
C/C & 0.231 & 23 & 12 & 9 & 5.125 & 1.325 & 0.185 \\
C/T & 0.472 & 35 & 12 & 17.5 & 8.75 & -1.859 & 0.063 \\
T/T & 0.296 & 24 & 12 & 9.5 & 5.375 & 1.078 & 0.281 \\
\hline
\end{tabular}

Fam\#, number of informative families; S, observed transmission of genotype to affected offspring; E, expected transmission under Mendelian inheritance; Var, variance S-E. P, two-tailed P value; significant $\mathrm{P}$ value $<0.05$;

**significant $\mathrm{P}$ value after Bonferroni correction $(\mathrm{P}<1.11 \mathrm{E}-4)$. 
Table 3. Associated haplotype compositions within NTRK3 in eating disorders

\begin{tabular}{|c|c|c|c|c|c|}
\hline SNPs & Haplotype* & $\begin{array}{c}\text { Haplotype } \\
\text { Frequency }\end{array}$ & Fam\# & $\begin{array}{c}\text { P Value } \\
\text { (FBAT) }\end{array}$ & $\begin{array}{c}\text { Power } \\
\text { (FBAT) }\end{array}$ \\
\hline rs7180942 - rs10520676 - rs2162266 & T_G_G & 0.488 & 143 & $7.86 \times 10^{-05 * *}$ & 0.17 \\
\hline rs7180942 - rs3784406 - rs2162266 & T_G_G & 0.480 & 127 & $0.0005^{* *}$ & 0.57 \\
\hline rs7180942 - rs3784406 - rs10520676 & T_G_G & 0.473 & 127 & $0.0007 * *$ & 0.68 \\
\hline rs3784406 - rs10520676 - rs2162266 & G_G_G & 0.535 & 134 & $0.0011^{* *}$ & 0.22 \\
\hline
\end{tabular}

Fam\# number of informative families; *For the heterozygous advantage model of inheritance, this column represents the haplotype that is carried in heterozygosis; **Significant after Bonferroni correction, 29 tests performed $(\mathrm{p}<0.0017)$. 
Table 4. Results of the TDT analyses for rs17033706 in NGF interaction with rs7180942 in NTRK3 in only families with rs7180942 heterozygous (protective genotypes) patients' and in only families with rs7180942 homozygous (risk genotypes) patients'. Interaction p value has been calculated using using a conditional logistic regression model

\begin{tabular}{|c|c|c|c|c|c|c|}
\hline & $\begin{array}{l}\text { rs17033706 } \\
\text { allele }\end{array}$ & $\begin{array}{c}\text { Allele } \\
\text { frequency }\end{array}$ & overtransmitted & $\mathbf{T}: \mathbf{U}$ & $\begin{array}{c}\text { Transmission } \\
\text { Odds ratio } \\
(95 \% \mathrm{CI}) \\
\end{array}$ & $\begin{array}{c}\text { Interaction } \\
\text { pval }\end{array}$ \\
\hline \multicolumn{7}{|l|}{$\begin{array}{c}\text { Only homozygous } \\
\text { (rs7180942) }\end{array}$} \\
\hline & A & 0.79 & A & $37: 10$ & $\begin{array}{c}13.69(3.43- \\
54.60)\end{array}$ & \multirow{5}{*}{$5.64 \times 10^{-5 * *}$} \\
\hline & G & 0.21 & & & & \\
\hline \multicolumn{6}{|l|}{$\begin{array}{l}\text { Only heterozygous } \\
\text { (rs7180942) }\end{array}$} & \\
\hline & A & 0.35 & G & $28: 15$ & $\begin{array}{c}3.45(1.03- \\
11.11)\end{array}$ & \\
\hline & G & 0.65 & & & & \\
\hline
\end{tabular}

** $\mathrm{p}$ value significant after Bonferroni correction $\mathrm{p}<4.85 \times 10^{-4}$; $\mathrm{T}: \mathrm{U}$ : transmitted versus non transmitted ratio. 


\section{Figure 1}

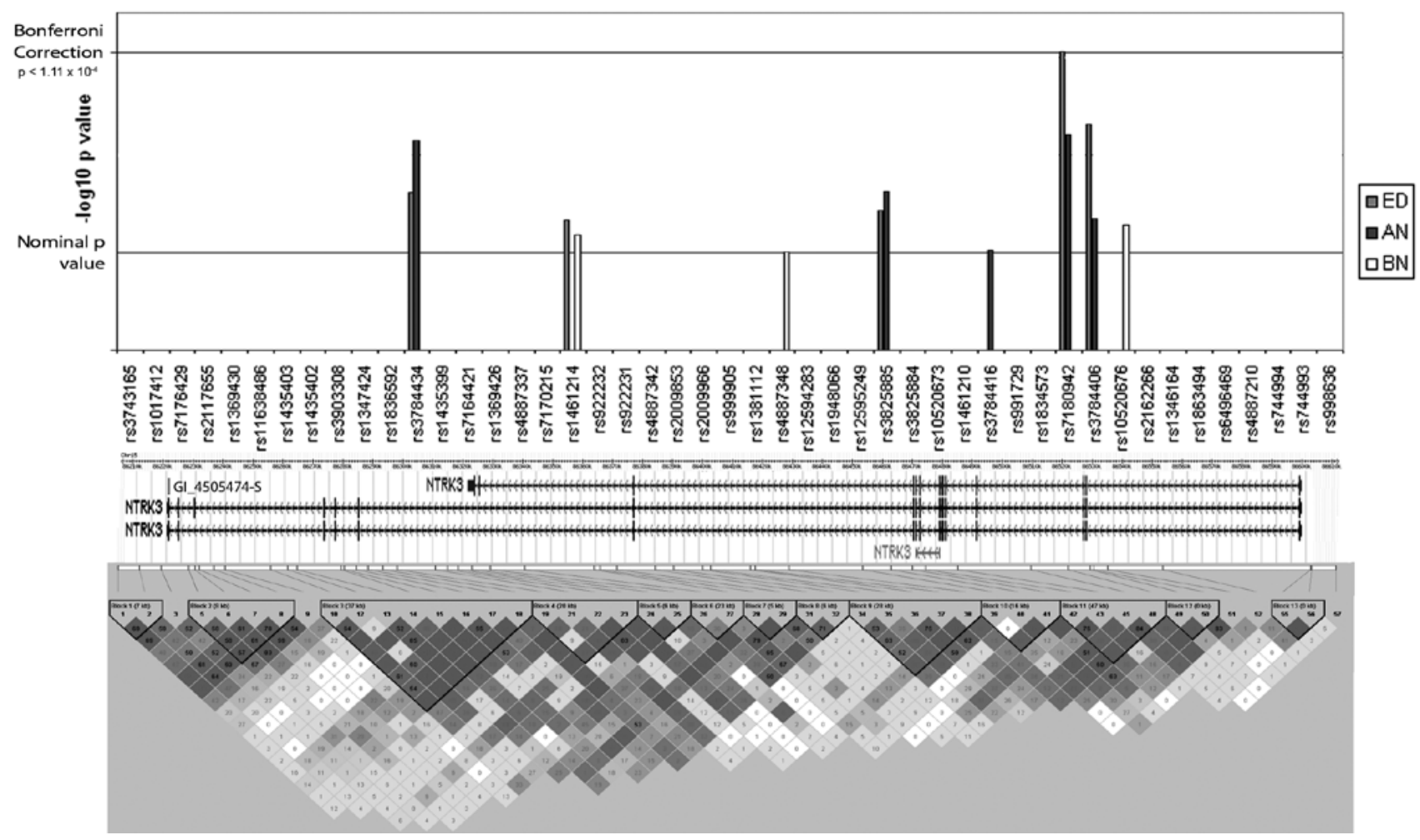

Figure 2

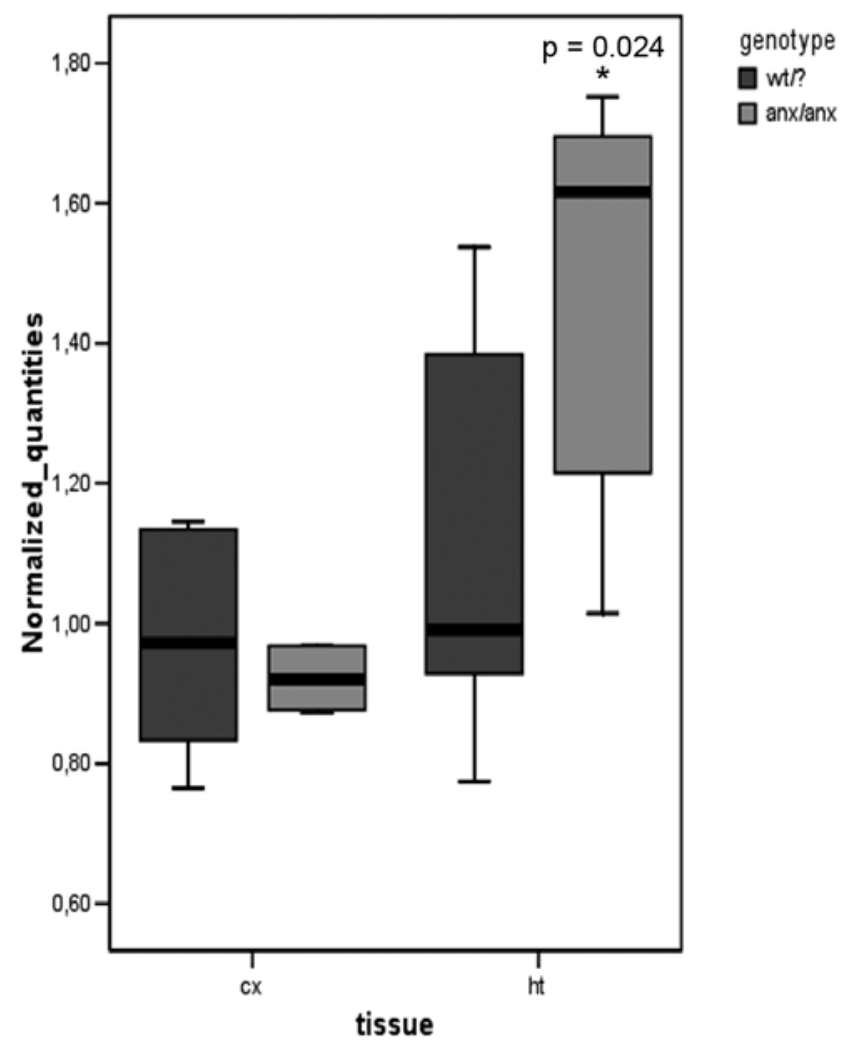




\section{Abbreviations:}

AN, anorexia nervosa; ANP, anorexia nervosa purging subtype; ANR, anorexia nervosa restricting subtype; BDNF, brain-derived neurotrophic factor; BN, bulimia nervosa; BNNP, non-purging bulimia nervosa subtype; BNP, binge-eating/purging bulimia nervosa; CIDI, Composite International Diagnostic Interview; CNTF, ciliary neurotrophic factor; CNTFR, ciliary neurotrophic factor receptor; Diagnostic and Statistical Manual of Mental Disorders, 4th edition, DSM-IV; DIGS, Diagnostic Interview for Genetic Studies; DIS-R, Diagnostic Interview Schedule-Revised; ED, eating disorders; EDNOS, ED not otherwise specified; EDNOS., ED not otherwise specified; LD, linkage disequilibrium; MAF, minor allele frequency; maxBMI, maximum body mass index; minBMI, minimum body mass index; NGFB, nerve growth factor; NGFR, p75, nerve growth factor; pan-neurotrophin receptor; NTF3, neurotrophins 3; NTF4/5., neurotrophin 4/5; NTRK1, neurotrophic tyrosine kinase, receptor, type 1; NTRK2, neurotrophic tyrosine kinase, receptor, type 2; NTRK3, neurotrophic tyrosine kinase, receptor, type 3; p75, NGFR, pan-neurotrophin receptor;nerve growth factor; SCID-I, Structured Clinical Interview for Mental Disorders, research version 2.0; SNP, single nucleotide polymorphism; UTR, untranslated region 